\section{Brain, Behavior and Evolution}

\title{
In Memoriam: Heinz Stephan (1924-2016)
}

Dr. Heinz Stephan, Scientific Member of the Max Planck Institute for Brain Research in Frankfurt am Main, passed away at the age of 91 years on January 3rd, 2016. The scientific community has lost a most influential comparative neurobiologist. Dr. Stephan's life-long research theme was the evolution of mammalian brains as a function of the animals' behavior and ecology. He has left a voluminous body of work that exhibits four principal characteristics:

- a sound theoretical background in comparative neurobiology

- a broad empirical grounding based on original data collected in the field

- a morphometric approach using brain and brain part volumes calculated from serial histological sections

- an allometric analysis of the brain data focused on the brain/body weight relationship

Overall, Stephan showed that brain evolution involves not simply a general increase or decrease of brain size, but selective alterations of brain parts that are under special demands or pressures in a given ecological niche (adaptive evolution). $\mathrm{He}$ focused on insectivores, primates and bats.

Stephan started the research, for which he became so famous, in 1956, by collecting brains of insectivores and other mammals, first in the east of Zaire, and then in many other countries around the world. Insectivores attracted his interest because they are said to present a basic, less differentiated condition. He demonstrated that some insectivores have relatively small brains, but that these are by no means undifferentiated. For example, the Malagasy tenrec, Echi- nops telfairi, has an extraordinarily large olfactory system. Moreover, the African otter shrews, Potamogale and Micropotamogale, the Malagasy water tenrec, Limnogale mergulus, and the European water shrew, Neomys fodiens, all have enlarged trigeminal systems, associated with numerous vibrissae that are used to detect prey under water [Stephan et al., 1991].

Besides insectivores, Stephan focused on primates, managing to collect many prosimian and simian brains from specimens in their natural habitat. The term "prosimian" again implied some undifferentiated, intermediate status on the way to simians and humans. Again, Stephan demonstrated that this assumption is only partly true by showing a remarkable diversity among prosimian brains. An outstanding example is Tarsius from South East Asia, a nocturnal insect hunter with a large brain that exhibits a greatly enlarged visual system; its primary visual cortex covers approximately one-fifth of the total neocortical surface and, in addition, shows an extremely differentiated laminar pattern. Stephan's data on simian brain and body size elucidated, for example, that the telencephala of folivorous Cercopithecidae (Colobinae) are smaller than those of frugivorous Cercopitecidae (Cercopithecinae). This pattern holds across all simians and still requires explanation.

In his studies of apes and Homo sapiens, Stephan was the first to demonstrate that the limbic system, particularly the hippocampus, is by no means reduced in humans, as was commonly assumed. The impression of a reduction is caused by the ex-

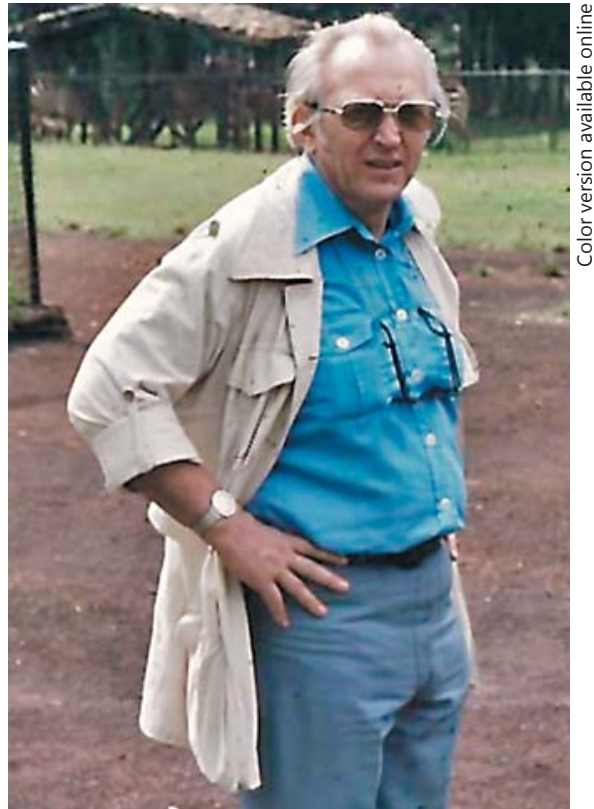

Heinz Stephan during the African expedition in 1979 (photo: Gerd Rehkämper).

tremely large neocortex of humans, in relation to which the hippocampus seems small. Stephan's allometric approach revealed a very different situation, namely an extraordinarily large human hippocampus. Because the limbic system is associated inter alia with affective behavior and memory, Stephan's results suggest that affective behavior is still important in humans, who are obviously not guided by solely "rational reasoning." Stephan's data also revealed that an outstandingly large neocortex

\section{KARGER}

(C) 2017 S. Karger AG, Base

Prof. Dr. rer. nat. Gerd Rehkämper

Institut für Anatomie I des Universitätsklinikums der Heinrich-Heine-Universität Düsseldorf, Geb. 22.04., Ebene 00, Raum 72, Moorenstrasse 5 DE-40225 Düsseldorf (Germany)

www.karger.com/bbe 
clearly sets humans apart from apes. When asked to explain this, Stephan often pointed to increased intraspecies competition, which is, today, what we essentially call the Machiavellian theory of human brain evolution.

Stephan was not uncritical about his own methods of comparison. The investigation of orangutans and gorillas, with their strong sexual dimorphism in body size, gave him the opportunity to demonstrate the limitations of the allometric approach. So did the investigation of species that have either undergone an evolutionary reduction in body size, such as Daubentonia, Miopithecus, and Hylobates, or a dramatic increase, such as the African elephant, Loxodonta, and whales. It is quite obvious that the low encephalization indices of such large mammals do not reflect their differentiated social behavior and cognitive abilities. Today, we refer to this idea as somatization versus encephalization.

Another mammalian order that attracted Stephan's interest was the bats (Chiroptera). Again, he went into the field to collect brains and body weight data all around the world. The Microchiroptera are sometimes said to be especially "intelligent" because of their echolocation system. Stephan showed that their neocortices are actually much smaller than expected but that, instead, their cerebellar structures are enlarged. This finding again demonstrated adaptive brain evolution, because the cerebellum is known to coordinate movements in threedimensional space, like bats experience during flight. Stephan even identified the limits of the extent to which mammalian brains can be reduced. Because the bamboo bat, Tylonycteris, roosts inside the hollow interior of bamboo plants (internodes), it has an extremely small and flattened skull that allows it to crawl into these small spaces. Stephan showed that the brain of $T y$ lonycteris is one of the smallest mammalian brains, perhaps outdone only by the largeeared tenrec Geogale aurita, which he studied as well.

Two very interesting groups of bats are the South American Phyllostomidae and Desmodontidae. Like Darwin's Galapagos finches, they have adapted to many ecological niches. Stephan demonstrated that this variety is reflected in the varied patterns of the volume of brain regions. Some impressive examples are the contrast between nectar-feeding bats, which behave like hummingbirds and have very large cerebella, and blood-eating vampire bats, which have well-developed olfactory regions. Stephan and his collaborators published this work on bats as a series of original papers, as well as in a three-volume opus magnum with Georg Baron and Heiko Frahm [Baron et al., 1996].

Stephan's data were used by many others, as reflected in the fact that his 1981 paper in Folia Primatologica [Stephan et al., 1981] has been cited 500 times. This subsequent work stimulated vivid discussions of Stephan's approach and yielded evolutionary conclusions that were sometimes controversial. Those who worked primarily with fossils and endocasts disliked Stephan's concept of the adaptive enlargement or shrinkage of brain parts, because it complicated the task of inferring brain anatomy from endocasts. Others advocated against using taxonomic groups to calculate allometric slopes and baselines, focusing instead on ecoethological entities such as dietary types. Stephan was open to this argument and used this concept in addition to his original approach. Similarly, Stephan recognized that adjusting his encephalization indices for basic metabolic rates caused many interspecies differences to become small or even to disappear. He was not happy about this, because metabolic rate-adjusted data should correspond with taxonomy and ecology, including dietary types. Interspecific diversity, for example in the neurobiology of the animals, is evident; he therefore questioned the use of basic metabolic rate as a "corrective." Another interesting development was the issue of developmental constraints on brain region sizes. Stephan himself did not actively take part in this evo-devo discussion, but we are sure that he would have been happy to learn how the brain of a given species develops into the adult form that is the target of natural selection. Finally, Stephan's approach to mammalian brain evolution provided an exemplary paradigm for the investigation of brain evolution in other vertebrate classes, an area of research that has blossomed in recent years.
Stephan, whose intellectual power was accompanied by a remarkably peaceful and balanced personality and mind, also produced another major monograph, namely "Allocortex" [Stephan, 1975], which he worked on for 20 years to summarize all that was known about the archi- and palaeocortex at the time. In addition, he amassed a brain collection that is outstanding for its size, diversity, and documentation. At the end of his active scientific life, he gave one of us (K.Z.) this collection, and it has continued to be used by many scientists. Housed at the Cécile and Oskar Vogt Institute of Brain Research at the University of Düsseldorf, the collection is still freely accessible.

We, who were fortunate to work closely with Heinz Stephan, have lost a wonderful mentor and friend. A strong memory of him will undoubtedly endure in our minds, as well as in the minds of numerous other comparative neurobiologists.

Gerd Rehkämper, Institute of Anatomy I, University of Düsseldorf, Germany

Leo Peichl, Max Planck Institute for Brain Research, Frankfurt am Main, Germany

Karl Zilles, Research Center Jülich and RWTH University, Aachen, Germany

\section{Acknowledgement}

We wish to thank Georg Striedter for encouragement and support to write this obituary.

\section{References}

Stephan H (1975): Allocortex. Handbuch der mikroskopischen Anatomie, vol IV/9. Berlin, Springer.

Stephan H, Frahm H, Baron G (1981): New and revised data on volumes of brain structures in insectivores and primates. Folia Primatol 35: $1-29$.

Stephan H, Baron G, Frahm HD (1991): Comparative Brain Research in Mammals, vol 1. Insectivora. New York, Springer.

Baron G, Stephan H, Frahm HD (1996): Comparative Neurobiology in Chiroptera, 3 vols. Basel, Birkhäuser. 